Ann. Zootech., I976, 25 (3), 4II-4I5.

NOTE

\title{
DIAGNOSTIC DU NOMBRE DE FETUS \\ CHEZ LA BREBIS PAR RADIOGRAPHIE AUX RAYONS X AVEC UN APPAREIL PORTABLE DE FAIBLE PUISSANCE
}

\author{
M. TISSIER et A. BRELURUT \\ avec la collaboration technique de P. CRoIzET \\ Station de Recherches sur l'Élevage des Ruminants, \\ Centre de Recherches de Clermont Ferrand I. N. R. A., \\ Theix, 63110 Beaumont
}

\section{RÉSUMÉ}

Nous avons décrit une technique de diagnostic du nombre de fretus chez la brebis entre go et I Io jours de gestation à l'aide d'un appareil portable de faible puissance. Il est nécessaire d'u tiliser des écrans renforçateurs aux " terres rares"; les paramètres techniques sont alors les suivants : voltage $=80 \mathrm{kV}$; intensité $=20 \mathrm{~mA}$; temps d'exposition $=\mathrm{I}, 5$ à 3 secondes suivant l'épaisseur abdominale de la brebis $\left(20\right.$ à $30 \mathrm{~cm}$ ). Pour 78 brebis radiographiées au ${ }_{10} 4^{\mathrm{e}}$ jour de gestation, lo diagnostic a été exact dans 92 p. Ioo des cas.

\section{INTRODUC'TION}

Plusieurs auteurs (BENzIE, I95I ; FORD et al., I963; Adran et BRown, I964; Wenham et RobINSON, I972) ont décrit une technique satisfaisante du diagnostic du nombre de foetus chez la Brebis grâce à la radiographie aux rayons X. Eintre le $70^{\mathrm{e}}$ et le $90^{\mathrm{e}}$ jour de gestation, le pourcentage de diagnostics corrects dépasse $95 \mathrm{p}$. Ioo (WENham et Robinson, I972). Cependant tous ces auteurs ont utilisé des matériels de forte puissance avec les paramètres d'exposition suivants : 65 à $80 \mathrm{kV}, 320 \mathrm{~mA}$ et 0,20 à $0,25 \mathrm{~s}$ soit 64 à $80 \mathrm{~mA} / \mathrm{s}$. Avec des appareils portables de faible puissance, la technique est rendue difficile du fait des durées d'exposition élevées (jusqu'à 
5 s) qui rendent gênants les moindres mouvements de la brebis, y compris les mouvellents respiratoires.

Je tels appareils peuvent cependant être utilisés avec des écrans renforçateurs "aux terres rares " (1), placés de part et d'autre du film, qui permettent de diviser les temps d'exposition approximativement par deux. Dans ces conditions, en réduisant au maximum les mouvements des animaux, nous avons pu obtenir des résultats satisfaisants.

\section{MATÉRIEL ET MÉTHODES}

Le générateur (le rayons $\mathrm{X}$ est un appareil portable ACOMA PX 30, avec 3 possibilités de réglagre clu voltage et de l'intensité : $60 \mathrm{kV}$-30 $\mathrm{mA} ; 80 \mathrm{kV}-20 \mathrm{~mA} ;$ 100 $\mathrm{kV}-\mathrm{I} 5 \mathrm{~mA}$.

Nous arons utilisé des cassettes de $30 \times 40 \mathrm{~cm}$, munies d'écrans renforçateurs "aux terres rares $n$, et une table orientable de $x_{\text {f }}{ }^{-}$f $^{\circ} \mathrm{cm}$ (voir photo) munie d'une grille focalisée (distance focale $70 \mathrm{~cm}$ ) et cl'un tiroir à cassette.

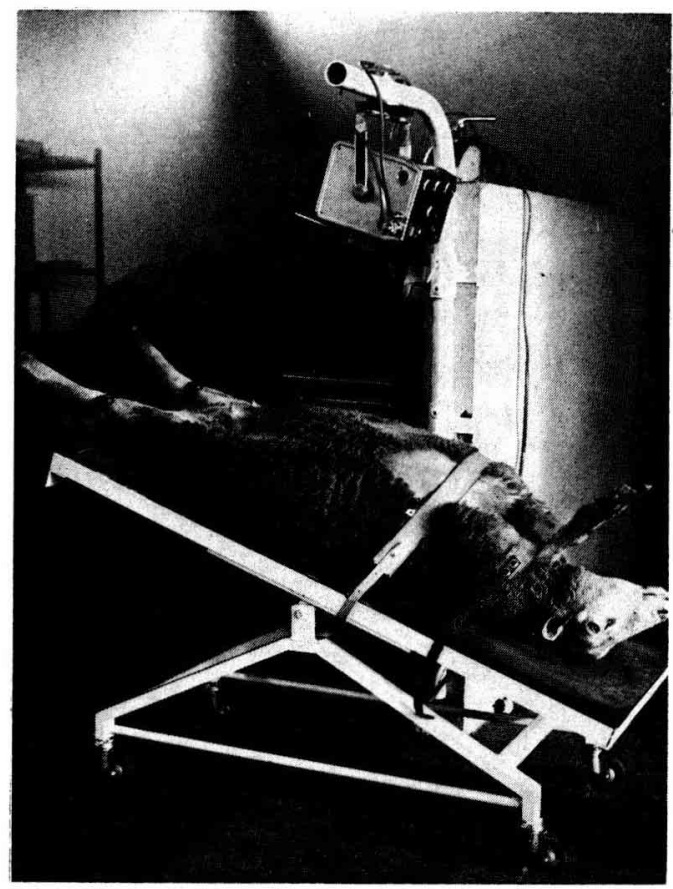

Fig. I. -... Brebis cn position sur table

La brebis (dont la laine doit ètre propre et sèche) est mise à jeun $2+$ h avant d'ètre radiographiée. On lui administre une faible dose de tranquillisant par voie intraveineuse environ I/4 d'heure avant l'opération.

La brebis est couchée sur la table en position dorso-ventrale, puis immobilisée par des sangles placées sur les pattes arrière et avant, ainsi que sur la poitrine, comme le montre la photographie. La table est ensuite penchée en avant, ce qui a pour but de faire avancer les réservoirs digestifs vers la cage thoracique et d'étaler l'utérus dans la cavité abdominale. On peut aussi comprimer l'abdomen à l'aide d'une bande de tissu pour diminuer l'épaisseur traversée par les rayons $\mathrm{X}$. De cette laçon ancun opérateur n'est nécessaire pour tenir l'animal.

(') Ces érans nut été récemment commercialisés par la Compagnie générale de Radiologie (C.(i.R.), sous la rubrigue $S$ l: , i base de terres rares luminophores (sels de (iadolinimu ot de Lanthanimm). 
Les paramètres tcchniques utilisés sont : distance source de rayons - film : $70 \mathrm{~cm}$; voltage : $80 \mathrm{kV}$; intensité : $20 \mathrm{~mA}$; durée d'exposition : de $\mathrm{I}, 5$ à 3 secondes suivant la taille de la brebis (épaisseur abdominale de $20 \mathrm{~cm}$ à $30 \mathrm{~cm}$ ).

Les films kodak Régulix $\mathrm{BB}_{54}$ ont été développés pendant $5 \mathrm{~mm}$ à $20^{\circ} \mathrm{C}$ dans le révélateur kodak LX 24. Pour 9 brebis sur les 95 radiographiées, nous avons dû faire un $2^{\mathrm{e}}$ film en raison cle la mauvaise qualité du premier, soit parce que l'animal avait bougé, soit parce que les paramètres d'exposition étaiont mal choisis.

\section{RÉSULTATS}

Nous avons appliqué cette technique à 95 brebis de race Limousine, afin de constituer des lots de brebis avec un agneau et 2 agneaux pour les besoins d'une expérience nutritionnelle. I,es dates de fécondation étaient connues. Les résultats des diagnostics effectués sont consignés dans le tableaur.

\section{TABLEAU I}

Comparaison entre le nombre d'agneaux nés et le nombre d'agneaux diagnostiqués par radiographie à différents stades de la gestation

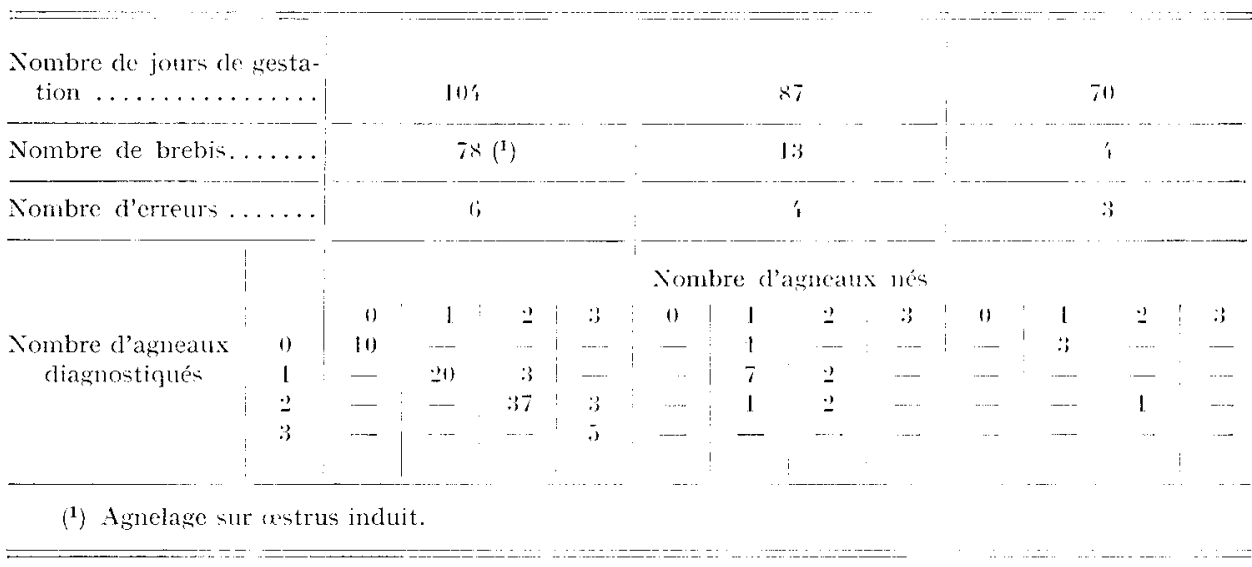

Les résultats sont satisfaisants au Io $^{\mathrm{e}}$ jour de gestation (photo 2 ), puisque le pourcentage de diagnostics erronés n'est que de $7,7 \mathrm{p}$. Ioo. Ils le sont beaucoup moins à des stades de gestation plus précoces, probablement en raison d'une ossification insuffisante du squelette des fœtus, mais le nombre de brebis étudié à ces stades est insuffisant.

Il faut remarquer que les diagnostics erronés correspondent généralement à une mauvaise position des brebis par rapport au film, ou à des clichés de mauraise qualité (flous, sous ou surexposés) qu'il aurait été nécessaire de refaire.

En conclusion, on peut dire qu'aux environs du roo jour de gestation on peut estimer le nombre de fotus de façon satisfaisante avec les appareils radiographiques portables de faible puissance. Les résultats devraient être encore améliorés grâce à quelques aménagements supplémentaires de notre installation, en particulier par la compression de l'abdomen avec une bande de tissu (ce qui diminue l'épaisseur tra- 


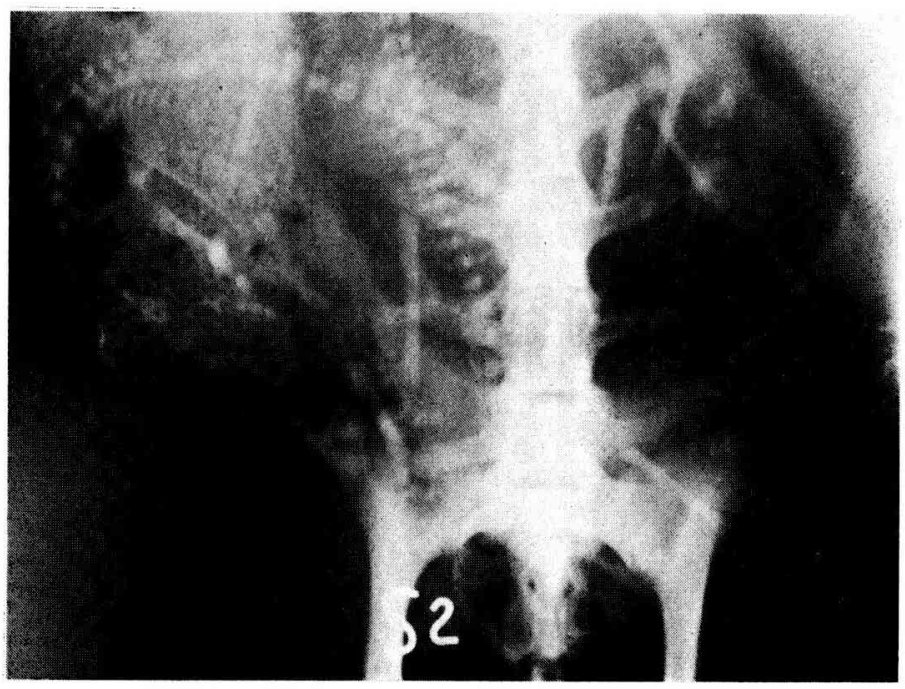

Fíg. 2. - Radiographie d'une brebis I.imousine portant 2 foetus à 704 jours do gestation. On pout voir un des foctus presque dans l'axe de la colonne vertébrale de la mère avec la tête dans le bassin, et lautre sur la ganche de la photographic.

versée par les rayons, et limite les mouvements de la mère et du foetus). La techinique est cependant plus difficile à mettre en ouvre qu'avec les appareils de forte puissance utilisés par les autres auteurs.

Rę̧u pou publication en avril 1976 .

\section{REMERCIEMENTS}

Nous remercions le personnel de la Compagnie générale de Radiologic, particulièrement MM. G. Bertail, J. Brisset et J.-F. Dufraise pour leurs conseils.

Nos remerciements vont également à G. BECHET pour les photographics.

\section{SUMMARY}

\section{X-RAY DIAGNOSIS OF FOETAL NUMBERS IN EWES BY MEANS OF A I.OW POWERED POR'TABLE APPARATUS}

A technique for X-ray diagnosis of foetal numbers in ewes between days go and ro of gestation by means of a low powered portable apparatus is described. Use of intensifying screens composed of "rare earthes " is required; the technical parameters were the following; voltage $=80 \mathrm{kV}$; intensity $20 \mathrm{~mA}$; exposure time --- $\mathrm{I}, 5$ to 3 seconds according to the abdominal thickness of the ewe $(20$ to $30 \mathrm{~cm}$ ). For $78 \mathrm{X}$-rayed eves on (lay 104 of gestation, the diagnosis was accurate in $92 \mathrm{p}$. Ioo of the cases. 


\section{RÉFÉRENCES BIBIIOGRAPHIQUES}

Ardran G. M., Brown T. H., 1964. X-ray diagnosis of pregnancy in sheep with special reference to the determination of the number of foetuses. J. Agric. Sci., Camb., 63, 205-207.

Benzie D., I95I. X-ray diagnosis of pregnancy in ewes. Br. Vet. J,, 10\%, 3-6.

Douglas S. W., Williamson H. D., r97o. Veterinary radiological interpretation, Heinemann, London. Ford E. J. H., Clark J. W., Gallup A. L., 1963. The detection of foetal numbers in sheep by means of X-rays. Vet. Rec., 75 (38), 958-960.

Wenham C., Robinson J.-J., I972. Radiographic pregnancy diagnosis in sheep. J. Agric. Sci. Camb., 78, $233-238$. 